INVESTIGACIÓN

\title{
APLICACIÓN DE LAS TIC EN EL ANÁLISIS Y PERCEPCIÓN DEL NIVEL DE VULNERABILIDAD DE EDIFICACIONES RESIDENCIALES DE LA CIUDAD DE CÚCUTA
}

\section{APPLY ICT IN THE ANALYSIS AND PERCEPTION OF THE RESIDENCIAL BUILDINGS VULNERABILITY LEVEL IN CUCUTA CITY}

\author{
MSc. Rodríguez Lizcano Adriana ${ }^{a}$, Ing. Dr. Roberto de la Cruz Centeno Lara ${ }^{\mathrm{b}}$ \\ ${ }^{a}$ Universidad Francisco de Paula Santander, Docente de Ingeniería Civil sede Cúcuta \\ Calle 7N No. 18E-09 Edif. Asturias Apto. 10-01, Cúcuta, Colombia, \\ adrianarodriguez@ufps.edu.co \\ ${ }^{\mathrm{b}}$ Universidad Autónoma de Yucatán, Estructuras y Materiales \\ roberto.centeno.lara@gmail.com
}

Fecha de recepción: 05-11-2016

Fecha de aprobación: 03-12-2016

\begin{abstract}
Resumen: Este artículo expone la sistematización de variables derivadas de un método científicamente reconocido para evaluar la vulnerabilidad sísmica (el de Benedetti-Petrini) en vivienda unifamiliar de un piso con Software de Información Geográfico (SIG) con lo que se generó la información en formatos gráficos y cartográficos que posibilita a cualquier usuario conocer el nivel de vulnerabilidad cualitativa de su vivienda, de la calle o del sector donde habita o pretende adquirir finca raíz, y proporciona insumos que permiten construir mapas de riesgo por efectos sísmicos que puedan actualizar o ajustar el Plan de Ordenamiento Territorial (POT). Los resultados de la investigación contribuirán a resolver los problemas constructivos propios de edificaciones de la zona y se convertirán en un pilar fundamental en la elaboración del Manual de Construcción Sismo Resistente de la ciudad de Cúcuta. Además, esto es un estudio que ayudará a las investigaciones que se adelanten sobre la configuración en planta y altura para reducir la vulnerabilidad sísmica de las edificaciones en base al análisis realizado.
\end{abstract}

Palabras clave: Análisis sísmico, Geo-referenciación, Índice de Vulnerabilidad, Método Benedetti-Petrini, Plan de Ordenamiento Territorial, Vulnerabilidad sísmica.

\begin{abstract}
This article presents the systematization of variables derived from a scientifically recognized method to evaluate the seismic vulnerability (Benedetti-Petrini's) in a one-story detached house with Geographic Information Software (GIS), generating information in graphic and Which allows any user to know the level of qualitative vulnerability of their dwelling, the
\end{abstract}


street or the sector where they inhabit or intend to acquire a real estate, and provides inputs that allow the construction of risk maps for seismic effects that can update or adjust the Territorial Planning Plan (TPP). The results of the research will contribute to solve the constructive problems of buildings in the area and will become a fundamental pillar in the construction of the Resistance Earthquake Construction Manual of the Cúcuta city. In addition, this is a study that will help advance research on the plant and height configuration to reduce the seismic vulnerability of buildings based on the analysis.

Keywords: Benedetti-Petrini method, Geo-referencing, Seismic analysis, Seismic vulnerability, Territorial Planning Plan, Vulnerability index.

\section{INTRODUCCIÓN}

Desde los orígenes de la sociedad el rol de la información ha ido cambiando significativamente, y en la actualidad es un hecho indiscutible que la información y conocimiento desempeñan un papel primordial para el desarrollo de esa misma sociedad. El volumen de conocimientos y de información disponible en la actualidad, así como su velocidad de actualización genera nuevos retos para su operación y manejo, entre los cuales se encuentra la capacidad para almacenarlos, catalogarlos y hacerlos disponibles en forma oportuna y a costos razonables al usuario final o en apoyo a la sociedad del conocimiento. Los retos técnicos se constituyen en mejoramiento y transmisión de la información.

La ciudad de Cúcuta y su área metropolitana necesitan incluir el componente de vulnerabilidad sísmica para edificaciones en sus planes de gestión de riesgo y el proyecto de microzonificación. Por tanto, se requiere implementar un procedimiento para aplicar las Tecnologías de la Información y la Comunicación (TIC) en el proceso de análisis cartográfico y presentación del nivel de vulnerabilidad de las edificaciones residenciales.
El gobierno Nacional de Colombia viene implementando desde hace más de 8 años una política de prevención de desastre y atención de emergencias. EI componente "riesgo" es fundamental para la reestructuración de los programas y estrategias que conforman los planes a nivel local y regional que se integran a su vez con los planes a nivel nacional. Entre los planes locales que conforman las políticas de prevención de desastres están los planes de gestión del riesgo, y entre las estrategias o insumos está la microzonificación sísmica de la ciudad de Cúcuta y su área metropolitana.

Para un estudio viable y factible es necesario el uso de métodos cuantitativos basados en un procedimiento más preciso como la metodología para evaluar la vulnerabilidad sísmica en edificios de mampostería estructural presentada por Ibáñez Palacios Diego (2015). Son extensiones propias de los procesos de análisis y diseño antisísmico recomendados por normas como la NSR-10, que tienen sus fundamentos en un modelo calibrado teniendo en cuenta el análisis dinámico inelástico de la edificación, permitiendo así conocer el comportamiento elástico y plástico paso a paso y el posterior 
colapso de la estructura, ya que requieren una alta complejidad de dicho modelo utilizado y la evaluación de la respuesta sísmica de la construcción.

La aplicación de las TIC en el proceso de enseñanza no es una novedad reciente en el mundo, sin embargo Colombia y más específicamente en la ciudad de Cúcuta se encuentran pocos estudios relacionados con este tema, por dicha razón, se profundiza en la investigación mediante procesos como los expuestos por Tamayo y Tamayo Mario.

La tecnología de la información y las comunicaciones tuvo una gigantesca y rápida evolución, y dada la actual tendencia que se esboza, innovaciones y facilidades muy interesantes continuarán surgiendo. Internet $\mathrm{y}$, en consecuencia, el correo electrónico y todo tipo de aplicaciones online, continuarán presentando y ofreciendo avances significativos, y servirán de base para el desarrollo de nuevos y prometedores sistemas.

\subsection{Formulación del problema}

¿Cómo desarrollar un procedimiento basado en las TIC acerca de las edificaciones residenciales para los tres barrios de la ciudad de Cúcuta, Norte de Santander, Colombia; que permita analizar, geo referenciar, cartografiar y difundir el conocimiento acerca del nivel de vulnerabilidad sísmica.?

Entre los departamentos de Colombia, Norte de Santander está ubicado según el mapa de amenaza sísmica en zonas de alto riesgo. Esto quiere decir que sus terrenos están constantemente influenciados por el choque de placas tectónicas y la liberación de energía a través de las fallas geológicas, de ahí que se produzcan los temblores.

Ingeominas (institución competente en el tema) reiteró que por su ubicación geográfica, Colombia es una de las regiones más propensas a sismos que hay en el mundo.

Ante esto los expertos, aconsejaron a los ciudadanos revisar si sus viviendas tienen estructuras sismo-resistentes; según ellos ésta es la medida de prevención más importante que hay que tomar ante un eventual temblor de tierra.

La probabilidad de que ocurran sismos de alto impacto en Cúcuta no es una teoría descabellada si se tiene en cuenta que, según los analistas, la ciudad podría experimentar movimientos de magnitud 8.0, en condiciones de extrema superficialidad de estos eventos.

Así lo determinan estudios de la escuela de Ingeniería Civil de la Universidad Francisco de Paula Santander, según los cuales la ciudad está en un riesgo mayor que otras localidades del país de sufrir graves afecciones por movimientos telúricos.

El mismo mapa de amenaza sísmica nacional del Servicio Geológico Colombiano ubica al departamento $\mathrm{y}$, por ende, a la ciudad en una zona de alta amenaza sísmica.

Cada una de las fallas geológicas divide la ciudad en tres sectores con características de suelo diferente: la ciudadela de Juan Atalaya, el valle del río Pamplonita y ciudadela de La Libertad. De acuerdo con el 
Ing. Carlos Flórez, magíster en ingeniería geotécnica y sísmica de la Universidad de Los Andes y docente de la Universidad Francisco de Paula Santander, la realidad de la ciudad en materia de este tipo de riesgo, es preocupante.

\section{METODOLOGÍA}

Siguiendo con el cumplimiento de los objetivos planteados, se ha recolectado información pertinente al estudio de las viviendas de tres barrios de la ciudad de Cúcuta (Claret, Chapinero y Comuneros) que cumplen con las características estipuladas, para posteriormente generar un análisis de vulnerabilidad enfocado en dichos resultados. Dichos sectores han sido seleccionados debido a que la problemática que se plantea es muy constante en esta parte de la ciudad. De esta forma, es frecuente ver construcciones mal edificadas que no cumplen con los lineamientos que estipula la Norma Sismo Resistente Colombiana.

Para formular un procedimiento de análisis del nivel de vulnerabilidad sísmica de las edificaciones residenciales de la ciudad de Cúcuta, que posteriormente será cartografiado como debe realizarse según el documento citado de Vulnerabilidad sísmica en centros urbanos de Maldonado Rondón, Esperanza \& Chío Cho Gustavo (2010) es necesario recurrir a un proceso informático que realice de forma más rápida y eficaz el procesamiento de los datos a fin de que la sistematización de las tareas manuales representen un ahorro en la variable tiempo y que la automatización permita reducir el margen de error humano y a su vez, se haga uso de las TIC que son la temática fundamental de este proyecto.

Para ello, una vez tomadas las encuestas y teniendo el soporte físico de los resultados, se procede con la programación de un asistente informático para el trabajo, en este caso, utilizando el recurso Microsoft Office Excel auxiliado con Visual Basic Avanzado (un complemento interno de Office que permite ingresar al código informático de las operaciones y reestructurarlo para generar procesos de carácter arbitrario, es decir, que puedan acomodarse según el interés de quien programa y producir un resultado acorde a la necesidad analítica actual, para este caso, la organización automática de la información por barrios, contemplando para cada vivienda su dirección y el conjunto de respuestas cualitativas a cada parámetro para ejecutar el análisis estadístico pertinente) de modo que sea indispensable para llevar a cabo dicha tarea solo el ingreso manual de los datos y el conocimiento suficiente el tecnologías de la información y procesamiento de datos expresado para la situación presente en manejo de programación Excel-VBA.

Para que la programación cumpla con la necesidad del usuario, debe intervenirse por medio de un asistente informativo abierto al código en un lenguaje de programación compatible con el medio en que se presentan los resultados (Microsoft Excel), tal como se ha mencionado, correspondiente al Visual Basic Avanzado (orientado a aplicaciones).

Por esta razón, se han generado rutinas atadas entre sí por medio de funciones iterativas cuyo funcionamiento es ordenado 
por el botón de comando de la plantilla de ingreso de datos que está al alcance del usuario.

Método Cualitativo (método del índice de vulnerabilidad).

El procedimiento de cálculo se basa en los resultados obtenidos en las once (11) preguntas del test calificadas como una letra entre $\mathrm{A}, \mathrm{B}$ y $\mathrm{C}$ asociadas cada una a un puntaje de 1 a 3 siendo 1 el valor correspondiente a la calificación $\mathrm{C}$ y el 3 a la A, de esta forma, "A" sugiere una alta vulnerabilidad sísmica y " $C$ " una baja. Además, cada parámetro del formulario asume un multiplicador diferente según la importancia del mismo denominado Peso y denotado por la letra "W", tal como lo estipula el Método de Benedetti, D. \& Petrini, V. (1984) en base al cual se ha realizado la estructura de obtención del IV. Dichos puntajes de 1 a 3 se referencian con la letra "P" y como se ha mencionado anteriormente, dependen de su clase de la "A" a la "C". El índice de vulnerabilidad "IV" se consigue ponderando cada subresultado como $\mathrm{PxW}$ y sumando el total de los 11 ítems.

Tabla 1. Descripción del parámetro con respecto al ítem.

\begin{tabular}{|c|c|c|}
\hline ITEM & PARAMETRO & $\mathbf{W}$ \\
\hline \multicolumn{3}{|c|}{ Organización del } \\
\hline & sistema sismo & \\
\hline 1 & resistente & 4 \\
\hline 2 & $\begin{array}{c}\text { Calidad del sistema } \\
\text { resistente }\end{array}$ & 1 \\
\hline 3 & $\begin{array}{l}\text { Resistencia } \\
\text { convencional }\end{array}$ & 1 \\
\hline 4 & $\begin{array}{c}\text { Posición del edificio y } \\
\text { cimentación }\end{array}$ & 1 \\
\hline 5 & $\begin{array}{c}\text { Diafragmas } \\
\text { horizontales }\end{array}$ & 1 \\
\hline 6 & $\begin{array}{c}\text { Configuración en } \\
\text { planta }\end{array}$ & 1 \\
\hline 7 & $\begin{array}{c}\text { Configuración en } \\
\text { elevación }\end{array}$ & 2 \\
\hline 8 & $\begin{array}{c}\text { Distancia máxima } \\
\text { entre los muros }\end{array}$ & 1 \\
\hline 9 & Tipo de cubierta & 1 \\
\hline 10 & $\begin{array}{l}\text { Elementos no } \\
\text { estructurales }\end{array}$ & 1 \\
\hline 11 & $\begin{array}{l}\text { Estado de } \\
\text { conservación }\end{array}$ & 1 \\
\hline
\end{tabular}

Fuente: Libro de vulnerabilidad sísmica en centros urbanos de Esperanza Maldonado Rondón y Gustavo Chío Cho.

Finalmente, para construir el mapa, es necesario disponer primeramente de un suministro cartográfico y actualizado de la ciudad, en especial de la zona que contiene a los 3 barrios en cuestión, validado por el Instituto Geográfico Colombiano Agustín Codazzi (IGAC), que es la entidad encargada de producir tal información oficial y la geo-referenciación básica de Colombia, además de elaborar el catastro nacional de la propiedad inmueble.

Como se explicó previamente ya han sido obtenidos valores de vulnerabilidad, en escala absoluta y porcentual, así como un color que ha sido asignado a cada valor mediante análisis matemáticos y gráficos, usando para ello la siguiente escala.

Tabla 2. Escala de colores de la muestra 


\begin{tabular}{ccc}
\hline No. ESCALA & COLOR & MUESTRA \\
\hline 0 & 15461375 & \\
1 & 14277119 \\
2 & 12961279 \\
3 & 11777023 \\
4 & 10461183 \\
5 & 9145343 \\
6 & 7961087 \\
7 & 6645247 \\
8 & 5460991 \\
9 & 4145151 \\
10 & 2960895 \\
11 & 1645055 \\
12 & 329215 \\
13 & 329215 \\
14 & 329215 \\
15 & 242 \\
16 & 222 \\
17 & 204 \\
18 & 184 \\
19 & 164 \\
\hline
\end{tabular}

Fuente: Colores en escala RGB del código de colores de Microsoft Excel.

Entre las variables halladas se tiene: Dirección, Índice de Vulnerabilidad (original, en escala de 1-10, valor porcentual), color (en código de colores de Microsoft Excel, en escala RGB). De tales variables puede prescindirse de dos de las escalas empleadas para el Índice de Vulnerabilidad y de una de las escalas de colores. Finalmente, se opta por manipular solo las variables Índice de Vulnerabilidad (Valor absoluto y porcentual) y Color (en código de colores de Excel). Aunque esta última no es genérica para transferir a AutoCAD se toma para reconvertirse en RGB mediante un procedimiento de programación VBA más exacto que entregará dicho código de colores con mayor precisión que la rutina matemática, dado que se hace a través de funciones propias de los compiladores gráficos, en un formato como el siguiente.

Tabla 3. Formato de análisis de cada vivienda

\begin{tabular}{|c|c|c|c|c|c|c|}
\hline DIRECCION: & & $M Z$ & No. & \# & . & \# \\
\hline ITEM & CLASE & $\mathrm{P}$ & $\mathrm{W}$ & $\mathrm{P} * \mathrm{~W}$ & & \\
\hline 1 & \multirow{11}{*}{$\frac{2}{\sqrt{2}}$} & \multirow{11}{*}{$\underset{\text { 学 }}{\stackrel{\Xi}{Z}}$} & 4 & \multirow{11}{*}{ 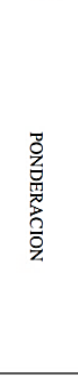 } & & \\
\hline 2 & & & 1 & & & \\
\hline 3 & & & 1 & & & \\
\hline 4 & & & 1 & & & \\
\hline 5 & & & 1 & & & \\
\hline 6 & & & 1 & & & \\
\hline 7 & & & 2 & & & \\
\hline 8 & & & 1 & & & \\
\hline 9 & & & 1 & & & \\
\hline 10 & & & 1 & & & \\
\hline 11 & & & 1 & & & \\
\hline $\mathrm{rv}-$ & $\Sigma(P * W)$ & & & RGB: & R-G-B & \\
\hline VALOR 1-10: & $10^{*}(\mathrm{IV} / 30-0,5)$ & & & COLOR: & \# & \\
\hline VALOR \%: & $100^{*}(\mathrm{IV} / 30-0.5)$ & & & MUESTRA & & \\
\hline
\end{tabular}

Fuente: Elaboración propia

\section{RESULTADOS}

Tabla 4. Rango de vulnerabilidad porcentual

\begin{tabular}{|c|c|c|c|c|c|c|}
\hline \multirow[b]{2}{*}{ BARRIO } & \multirow[b]{2}{*}{ TOTAL } & \multicolumn{5}{|c|}{$\begin{array}{l}\text { RANGO VULNERABILIDAD } \\
\text { PORCENTUAL }\end{array}$} \\
\hline & & $\begin{array}{l}0 \%- \\
20 \% \\
\end{array}$ & $\begin{array}{l}20 \%- \\
40 \% \\
\end{array}$ & $\begin{array}{l}40 \%- \\
60 \% \\
\end{array}$ & $\begin{array}{l}60 \%- \\
80 \% \\
\end{array}$ & $\begin{array}{l}80 \%- \\
100 \%\end{array}$ \\
\hline CHAPINERO & 141 & 13 & 38 & 38 & 38 & 14 \\
\hline CLARET & 66 & 1 & 28 & 28 & 5 & 4 \\
\hline COMUNEROS & 129 & 6 & 27 & 52 & 28 & 16 \\
\hline TOTAL & 336 & 20 & 93 & 118 & 71 & 34 \\
\hline
\end{tabular}

Fuente: Elaboración propia.

Tabla 5. Análisis estadísticos de la calificación de vulnerabilidad sísmica. 


\begin{tabular}{ccccc}
\hline BARRI0 & CHAPINER0 & CLARET & CONUNEROS & TOTAL \\
\hline TOTAL & 141 & 66 & 129 & 336 \\
\hline $0-20$ & 13 & 1 & 6 & 20 \\
$20-40$ & 38 & 28 & 27 & 93 \\
$40-60$ & 38 & 28 & 52 & 118 \\
$60-80$ & 38 & 5 & 28 & 71 \\
$80-100$ & 14 & 4 & 16 & 34 \\
\hline
\end{tabular}

Fuente: Elaboración propia.

Tabla 6. Estadística descriptiva para la muestra en general.

\begin{tabular}{lr}
\hline \multicolumn{2}{c}{ GENERAL } \\
\hline Media & 67,2 \\
Error típico & 18,1532366 \\
Mediana & 71 \\
Moda & 50 \\
Desviación estándar & 40,5918711 \\
Varianza de la & \\
muestra & 1647,7 \\
& - \\
Curtosis & 1,88507992 \\
Coefíciente de & \\
asimetria & 0,04096991 \\
Rango & 98 \\
Mínimo & 20 \\
Máximo & 118 \\
Suma & 336 \\
Cuenta & 5 \\
Nivel de confianza & 50,401465 \\
(95,0\%)
\end{tabular}

Fuente: Elaboración propia.

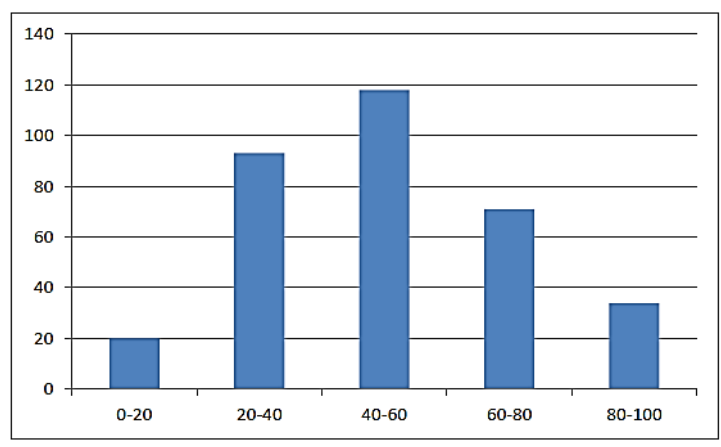

Figura I. Diagrama del Análisis estadístico de la calificación de vulnerabilidad sísmica de todos los barrios. Fuente: Elaboración propia.

\section{CONCLUSIONES}

Las Tecnologías de la Información y la Comunicación (TIC) son muy importantes en el desarrollo de cualquier proyecto por medio de la implementación de procesos modernos en la manipulación y procesamiento de datos.

La adopción de estas metodologías para el cálculo del Índice de Vulnerabilidad representa un aumento significativo en la eficiencia de dicha valoración de riesgo y estimula el crecimiento informático de las entidades que lo operen, basándose en métodos numéricos para Ingeniería como los citados en los documentos de los autores Yépez, F.; Barbat, H.; Canas, J.

El conocimiento de los índices de vulnerabilidad sísmica de las estructuras es indispensable para la adecuación de los planes de contingencia garantizando la seguridad de los habitantes, en especial si se trata de construcciones antiguas como es comentado en el aparte de la Introducción al Estudio de la Vulnerabilidad Sísmica de 
Edificios Históricos de Arango Gonzales José Ramón. (2003), para el caso presente de los tres barrios, el resumen de resultados de la investigación que se presenta en la tabla 4.

\section{FINANCIACIÓN}

la investigación es financiada por la misma fuente, pero se agradece a la Universidad Francisco de Paula Santander por la prestación de las instalaciones para el procesamiento de datos y la disposición de pasantes de ingeniería para la toma de la información pertinente para el estudio, mediante la modalidad estadística de encuestas.

\section{BIBLIOGRAFÍA}

\section{IBÁÑEZ PALACIOS DIEGO. (2015) Metodología para evaluar la vulnerabilidad sísmica en edificios de mampostería estructural.}

YEPEZ, F.; BARBAT, H.; CANAS, J. "Riesgo, peligrosidad y vulnerabilidad sísmica de edificios de mampostería". Barcelona: Centro Internacional de Métodos Numéricos en Ingeniería (CIMNE), 1995. ISBN 84-87867-50-2.

ARANGO GONZALES JOSÉ RAMÓN. (2003) Introducción Al Estudio De La Vulnerabilidad Sísmica De Los Edificios Históricos De Granada.

TAMAYO Y TAMAYO MARIO. El Proceso de la Investigación Científica. Limusa Noriega Editores. Tercera Edición. México, 1999. Págs.: 54-55.
BENEDETTI, D. \& PETRINI, V. (1984). Sulla vuinerabilitá sísmica di edififi in muratura. Proposte de método di valutazione. Roma: Lindustria delle construzioni.

MALDONADO

RONDON, ESPERANZA; CHIO CHO GUSTAVO (2010). Vulnerabilidad sísmica en centros urbanos Segunda Edición. Bucaramanga, Colombia. Universidad Industrial de Santander. 